\title{
Train velocity estimation method based on an adaptive filter with fuzzy logic
}

\author{
Petr Pichlík, Jiří Zděnek*
}

\begin{abstract}
The train velocity is difficult to determine when the velocity is measured only on the driven or braked locomotive wheelsets. In this case, the calculated train velocity is different from the actual train velocity due to slip velocity or skid velocity respectively. The train velocity is needed for a locomotive controller proper work. For this purpose, an adaptive filter that is tuned by a fuzzy logic is designed and described in the paper. The filter calculates the train longitudinal velocity based on locomotive wheelset velocity. The fuzzy logic is used for the tuning of the filter according to actual wheelset acceleration and wheelset jerk. The simulation results are based on real measured data on a freight train. The results show that the calculated velocity corresponds to the actual train velocity.
\end{abstract}

K e y w o r d s: slip control, adhesion phenomenon, fuzzy logic, adaptive filter, train velocity

\section{Introduction}

Some types of train slip controllers or other controllers need to know the train velocity for its proper work. The train velocity is typically determined from the train wheelsets velocity. For the purpose, it is required to distinguish between driven or braked wheelsets and nondriven wheelsets. The actual train velocity could be determined from a non-driven wheelset only because the driven wheelset has higher circumference velocity than its longitudinal velocity and the braked wheelset has its circumference lower than longitudinal velocity. Every wheelset is braked, and every locomotive wheelset is driven when the train is hauled by a locomotive. Therefore, the velocity measured by locomotive wheelsets is different from the actual train velocity during locomotive haul or brake. The actual locomotive velocity could be measured only during train coasting.

Although it is not appropriate, the train velocity is typically determined from the wheelset shafts velocity that is measured by incremental encoders that are placed on wheelsets shaft or the motors shafts. For this purpose, it is no difference between positions of incremental encoder because the motor shaft and wheelset are connected by the transmission with fixed gear ratio. The difference between the wheel circumference velocity and its longitudinal velocity is caused by a phenomenon called a slip velocity in traction or skid velocity in braking. The value of the slip velocity could be up to $2 \mathrm{~km} / \mathrm{h}$ under normal operation conditions [1], but in some undesirable cases, the velocity could be more times higher. The value of the slip velocity depends on the applied force between wheels and rails and a slip characteristic shape. The shape of the characteristic depends on a rail condition, temperature and wheelset velocity. The characteristic is nonlinear and has a maximal value. When the characteristic maximal value is overstepped the slip velocity can rapidly increase, and the determined train velocity is distorted.

To eliminate the high value of the slip or skid velocity, the slip controllers are used, and they are described in the literature for trains [2], [3] and automotive [4]. The train longitudinal velocity is typically derived from the locomotive driven wheelsets velocity, or when it is possible by velocity measuring on a non-driven wheelset. Sometimes are used Doppler radars or GPS devices.

There is the estimated difference between velocity measured on driven wheelsets and the actual Strain velocity in the paper. A mathematical description of a nonlinear adaptive filter is presented, and fuzzy logic is described, too. Calculated wheelset acceleration and wheelset jerk are inputs to the fuzzy logic control part. Finally, the simulation results are presented. The simulation input data are based on measurement from real electric locomotive with a freight train. The results are focused on cases when a high value of the slip velocity occurs.

\section{Method}

\subsection{Wheel velocity and train velocity}

The most common way to determine train velocity is to calculate the velocity from the measured wheelsets velocity. When the wheel is driven, or braked there is a difference between the train velocity and a wheel circumference velocity. The difference is called a slip velocity or skid velocity respectively. The slip velocity is defined as

$$
v_{\mathrm{S}}=v_{\mathrm{C}}-v_{\mathrm{L}}
$$

* Dept. of Electric Drives and Traction, Czech Technical University in Prague, Faculty of Electrical Engineering, Technická 2, 166 27 Prague, Czech Republic, pichlpet@fel.cvut.cz, zdenek@fel.cvut.cz 


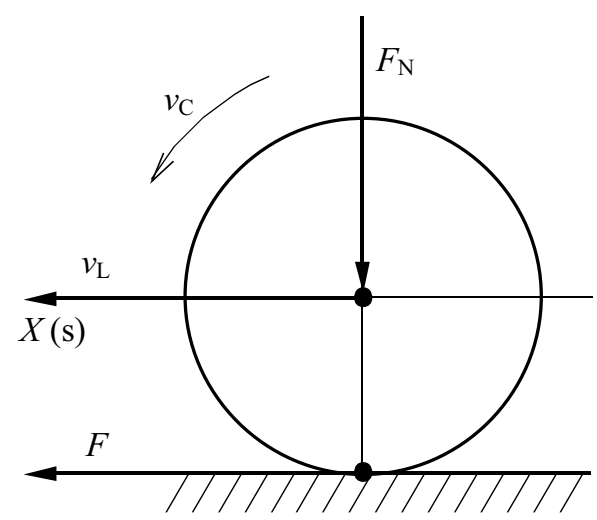

Fig. 1. Forces and velocities on a driven wheel

where $v_{\mathrm{S}}$ is the slip velocity, $v_{\mathrm{C}}$ is the wheel circumference velocity, and $v_{\mathrm{L}}$ is the longitudinal wheel velocity.

The relations between the driven wheel longitudinal velocity and the wheel circumference velocity are shown in Fig. 1. A tractive effort $F$ and normal force $F_{\mathrm{N}}$ is applied on the wheel.

The train longitudinal velocity could be determined by several ways. The common way is to estimate the velocity from wheelset velocity by some averaging or using a velocity of the slowest wheelset as a train velocity, but this way does not ensure determination of the train actual velocity. The methods could fail when all wheels have a high value of the slip or skid velocity, especially when all wheelsets has a high value of the slip velocity simultaneously. The electric multiple unit could have some non-driven wheelsets, and its velocity could be used for the train velocity calculation when the train is in traction; but, during braking all wheelsets are braked, and this solution does not work. The train velocity estimation is based only on locomotive measurement devices. The estimation could be improved by proper software algorithms. There are described many systems for an automotive industry that could be used for a train too in the literature. These algorithms include an adaptive filter [5] or on fuzzy logic connected with some filtration type or estimation method. For this purpose, the connection of the fuzzy logic and a Kalman filter [6], [7] is typically used. In some cases, the incremental encoder could be replaced by estimated velocity by sensorless vector control [8]. However, it is still measured the wheel circumference velocity in principle. Another way is to use the Doppler radars [9], [10] but this solution requires installation of additional radars on the vehicle. The third way is to use a GPS. The GPS can have outages in some areas and use of the GPS has to be connected with sensors fusions because the GPS could not be able to provide sufficient accuracy [11].

\subsection{Principle of the method}

The basic block diagram of proposed method is shown in Fig. 2. The block diagram contains four main parts that are minimal or maximal wheelsets velocity calculation,

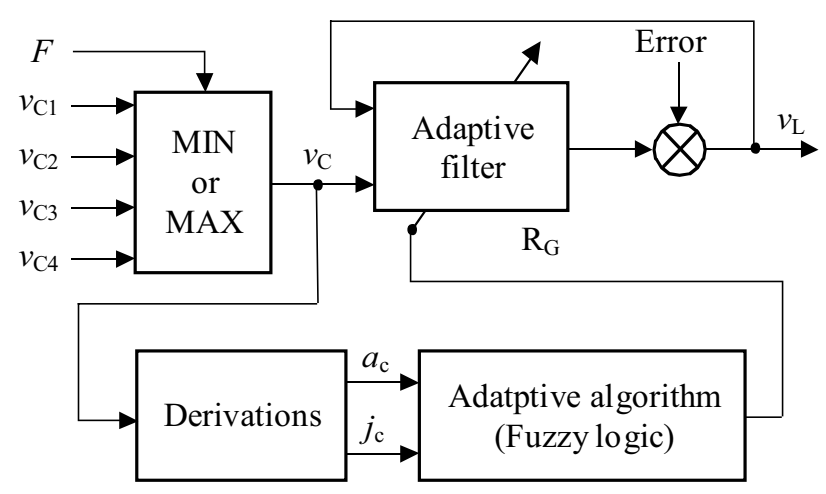

Fig. 2. Block diagram of the train velocity calculation

acceleration and jerk calculation, a fuzzy logic as adaptive algorithm and an adaptive filter.

The purpose of this method is to estimate the train longitudinal velocity $v_{\mathrm{L}}$ from the wheelsets velocity. The method uses measured wheelset velocity from all available wheelsets that are marked $v_{\mathrm{C} 1}$ to $v_{\mathrm{C} 4}$. From the velocity values, the minimal velocity is calculated when the wheelsets are driven and the maximal one when the wheelsets are braked. The maximal or minimal function is set according to the value of required traction effort $F$. The maximal or minimal velocity is marked as calculated wheelset circumference velocity $v_{\mathrm{C}}$. The wheelset circumference velocity value is not the same as the train velocity due to the slip velocity or skid velocity. The calculated wheelset circumference velocity is led to the adaptive filter. The adaptive filter has adjustment parameter $R_{\mathrm{G}}$. The parameter should correspond to the train acceleration to ensure the proper filter work. Therefore, the parameter is tuned by an adaptive algorithm that is based on fuzzy logic. The fuzzy logic inputs are the wheelset acceleration $a_{\mathrm{c}}$ and the wheelset jerk $J_{\mathrm{C}}$. The values of calculated acceleration and jerk contain high noise level. The noise represents the actual state of the system that is caused $e g$ by the train acceleration, track slope going through a switch or by some unevenness on track. When a non-adaptive filter is used, the acceleration has to be filtered to remove the noise.

The acceleration and jerk are calculated from wheel velocity. However, the actual velocity depends on the wheels diameter. The diameter is not constant, and its value decreases during the train operation over a long time period. The locomotive computer calculates typically the wheelset velocity for the maximum wheel diameter. Therefore, the measured velocity proportionally increases with decreasing diameter. The velocity could be correctly calculated when the wheel diameter is correctly set that needs periodic readjustment [12] the wheel diameter in the locomotive computer. Therefore, the train velocity could not be calculated as an actual train velocity by this method, and its value depends on the wheel diameter too. For some purpose, the actual value of the train velocity is not required, but relative velocity to the 


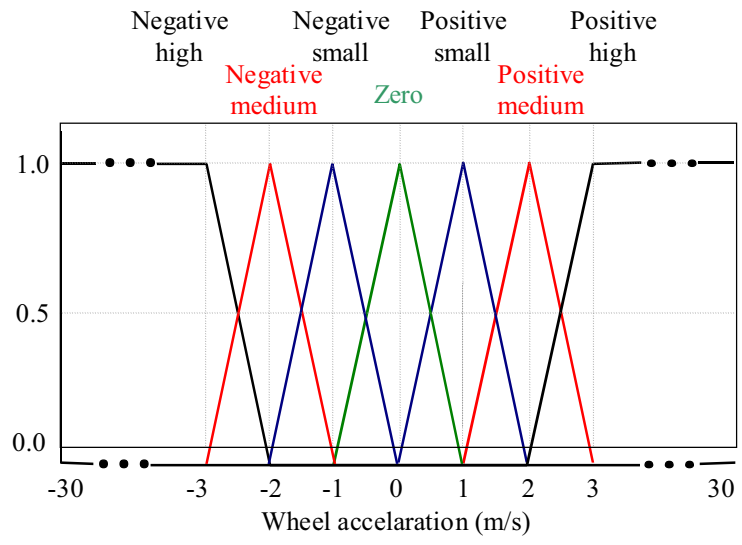

Fig. 3. Input membership function of wheel acceleration

wheel velocity is sufficient. An example of the slip controllers that needs to know the train velocity but not actual velocity are based on a disturbance observers [13], [14] because when the diameter is changed the both velocities also change, with the same ratio.

There are two types of errors that can occur in the system. The first error type is included in the measured wheel velocity. The velocity measurement method causes the errors. The adaptive filter takes into account the errors. The second error types cannot be distinguished from the wheel velocity. The main error in this group is a wheel diameter error. These error types are covered in Error input in Fig. 2.

\subsection{Adaptive filter}

The train velocity could be calculated from the equation of motion

$$
M \frac{\mathrm{d} v_{\mathrm{T}}}{\mathrm{d} t}=F-F_{\mathrm{L}}
$$

Where $v_{\mathrm{T}}$ is the train velocity, $F$ is the locomotive tractive effort and $F_{\mathrm{L}}$ is load force that is created by load forces and $M$ is a train mass.

The load forces are variable in time and position, and it is difficult to determine them. Some methods enable the load forces estimation. The methods are based on state observers [15], and the methods require precise system model and its parameters. The actual train velocity could be calculated when a ratio of forces and mass is replaced by a general acceleration in the (2). The problem is in the acceleration changes. If the acceleration is calculated from the wheelset acceleration, the calculated train velocity is not the actual train velocity but it is the wheelset velocity and does not correspond to the train velocity due to the slip or skid velocity. If the acceleration value is properly tuned the train velocity could be calculated. The task could be solved by a nonlinear adaptive filter. The used filter uses only a minimum or a maximum of measured wheelset velocity for estimation and one adjustment parameter [5], [16]. The adjustment parameter is based on wheelset acceleration, and its value is tuned

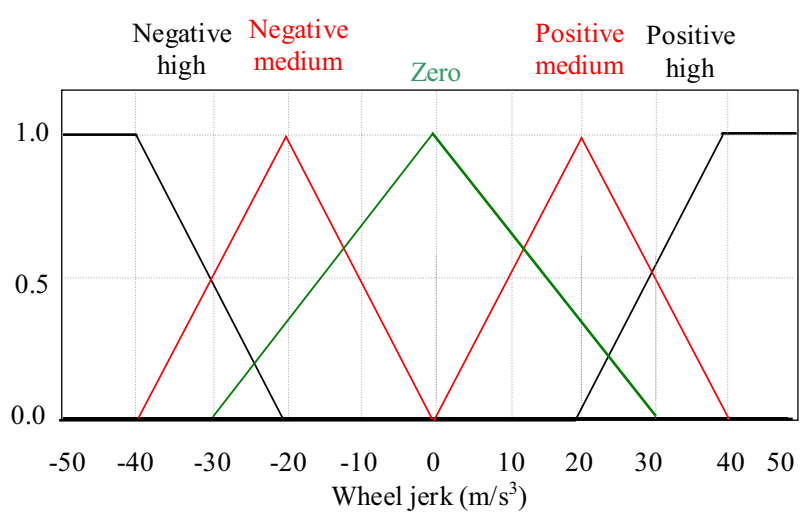

Fig. 4. Input membership function of wheel jerk

by the fuzzy logic. The filter is described as

$$
\frac{\mathrm{d} v_{\mathrm{L}}}{\mathrm{d} t}=-R_{G} \times \operatorname{sat}\left(v_{\mathrm{L}}-v_{\mathrm{C}}, d\right)
$$

where $v_{\mathrm{L}}$ is an estimated train longitudinal velocity, $v_{\mathrm{C}}$ is the calculated wheelset circumference velocity, $R_{\mathrm{G}}$ is an adjustment parameter of filter sensitivity, and $d$ is saturation function parameter.

The saturation function determines the sign of acceleration and enables the transition from one sign state to the second state smoothly. The saturation function could be replaced by a sign function. However, the sign and hysteresis function do not enable the smooth transition from one state to the second one. The sign function can cause the filter numerical oscillations [5]. The saturation function is defined as

$$
f(x, d)=\operatorname{sat}(x, d)=\left\{\begin{array}{rl}
1 & x>d \\
-1 & x<d \\
\frac{x}{d} & \text { else }
\end{array}\right.
$$

When the filter is not properly set it causes that the vehicle velocity $v_{\mathrm{L}}$ will converge to the wheel circumference velocity $v_{\mathrm{C}}$ in steady state. This feature could cause some inaccuracy because the vehicle velocity is different by slip or skid velocity from longitudinal wheel velocity when the wheels transmit a force to a rail. The value of $R_{\mathrm{G}}$ limits train velocity change rate, and the value has to correspond to the actual train acceleration. However, the value of acceleration can significantly change for a loaded train and an empty train as well as a freight train and passenger train. The proper filter function depends on the value of the filter adjustment parameter, and it is appropriate to change the value according to immediate conditions.

\subsection{Fuzzy logic}

The fuzzy logic is used for tuning the adaptive filter adjustment parameter of filter sensitivity $R_{\mathrm{G}}$. A non-tuned variant of the adaptive filter has the adjustment parameter with a constant value. For the train longitudinal velocity estimation, the parameter cannot be constant due 


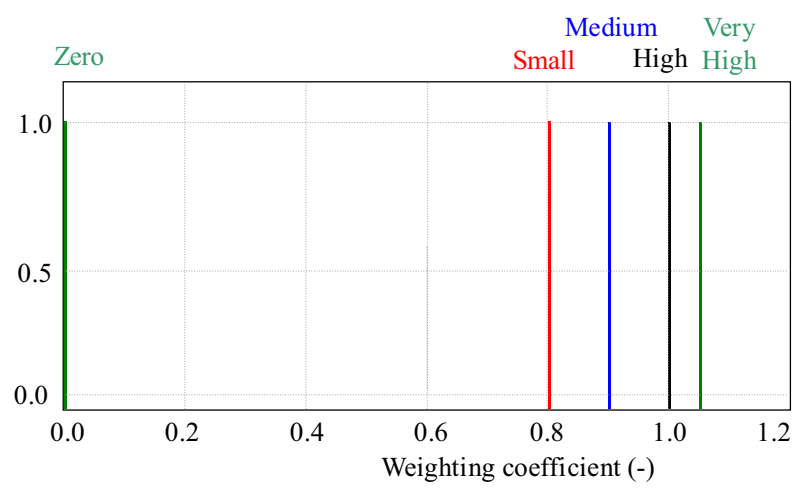

Fig. 5. Membership function of weighting coefficient

\begin{tabular}{|c|c|c|c|c|c|c|c|c|c|}
\hline \multirow{3}{*}{\multicolumn{3}{|c|}{ Fuzzy Logic Rules }} & \multicolumn{7}{|c|}{ Acceleration } \\
\hline & & & \multicolumn{3}{|c|}{ Positive } & \multirow{3}{*}{$\frac{\stackrel{0}{0}}{\mathrm{~d}}$} & \multicolumn{3}{|c|}{ Negative } \\
\hline & & & \multirow{2}{*}{$\frac{\frac{5}{503}}{Z}$} & \multirow{2}{*}{$\frac{\underbrace{\mathrm{Z}}_{\mathrm{Z}}}{\sum_{\mathrm{Z}}^{\mathrm{d}}}$} & \multirow{2}{*}{\begin{tabular}{|l}
$\overline{\bar{\pi}}$ \\
岂 \\
$\mathrm{Z}$
\end{tabular}} & & \multirow[t]{2}{*}{$\begin{array}{l}\overline{\bar{\tau}} \\
\bar{y}\end{array}$} & \multirow{2}{*}{ 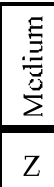 } & \multirow{2}{*}{$\frac{\frac{\sigma}{G}}{\mathrm{Z}}$} \\
\hline \multirow{5}{*}{$\stackrel{y}{\bar{J}}$} & $\stackrel{0}{i}$ & High & & & & & & & \\
\hline & 2 & Medium & $\mathrm{Z}$ & $\mathrm{S}$ & M & $\mathrm{H}$ & $\mathrm{M}$ & $\mathrm{S}$ & Z \\
\hline & \multicolumn{2}{|r|}{ Low } & $\mathrm{H}$ & $\mathrm{H}$ & $\mathrm{H}$ & VH & $\mathrm{H}$ & $\mathrm{H}$ & $\mathrm{H}$ \\
\hline & \multirow{2}{*}{ 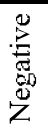 } & Medium & $\mathrm{Z}$ & $\mathrm{S}$ & M & $\mathrm{H}$ & M & $\mathrm{S}$ & Z \\
\hline & & High & Z & $\mathrm{Z}$ & Z & $\mathrm{H}$ & $\mathrm{Z}$ & $\mathrm{Z}$ & Z \\
\hline
\end{tabular}

Fig. 6. Fuzzy logic rules

to the slip velocity that can have high change rate due to the slip phenomenon. In [5], there is proposed method that can set the parameter $R_{\mathrm{G}}$ according to really calculated wheel acceleration. However, the parameter $R_{\mathrm{G}}$ is set when the estimated train velocity has the same value as wheel circumference velocity. The designed fuzzy logic enables to change the parameter $R_{\mathrm{G}}$ in every calculation step to achieve a match between estimated velocity and actual velocity. The fuzzy logic inputs are the calculated wheel acceleration and the wheel jerk. The fuzzy logic output is a weighting coefficient $R_{\mathrm{F}}$. The $R_{\mathrm{G}}$ parameter is calculated as

$$
R_{\mathrm{G}}=a_{\text {wheel }} \times R_{\mathrm{F}},
$$

where $a_{\text {wheel }}$ is the wheelset acceleration.

The input membership function of the wheel acceleration is shown in Fig. 3 and the membership function of the wheel jerk is shown in Fig. 4. The used Fuzzy Interference System (FIS) is of the Sugeno type. Therefore, the output membership function contains only singletons. The output membership function is shown in Fig. 5 .

When the acceleration and jerk have zero value or value near the zero, the output value of this coefficient has to be $R_{\mathrm{F}}=1$. When the acceleration and the jerk have a small value, which is different from zero. The output value decreases due to fuzzy logic rules. In the case, it is required the output value to be $R_{\mathrm{F}}=1$. Therefore, the Very High value on output membership function has value 1.1 .

When the slip velocity is low and when the velocity changes slowly, as it is typical for the freight trains, the fuzzy logic can work with the acceleration value to estimate the parameter $R_{\mathrm{G}}$. The high value of the slip velocity is characterised by the high value of the acceleration. The jerk is calculated as the derivation of the wheel acceleration. Therefore, the jerk does not contain information about the acceleration trends and provides the information about acceleration change rate only. This information enables to determine the beginning of the slip increase. The jerk moreover can detect any change of acceleration and therefore, it can detect any acceleration change faster than detection based on acceleration only.

The fuzzy rules are summarised in Fig. 6. The fuzzy logic output value is Very High for small or zero acceleration and jerk, and the output value decreases with increasing of the acceleration and jerk values. The fuzzy logic surface is shown in Fig. 7.

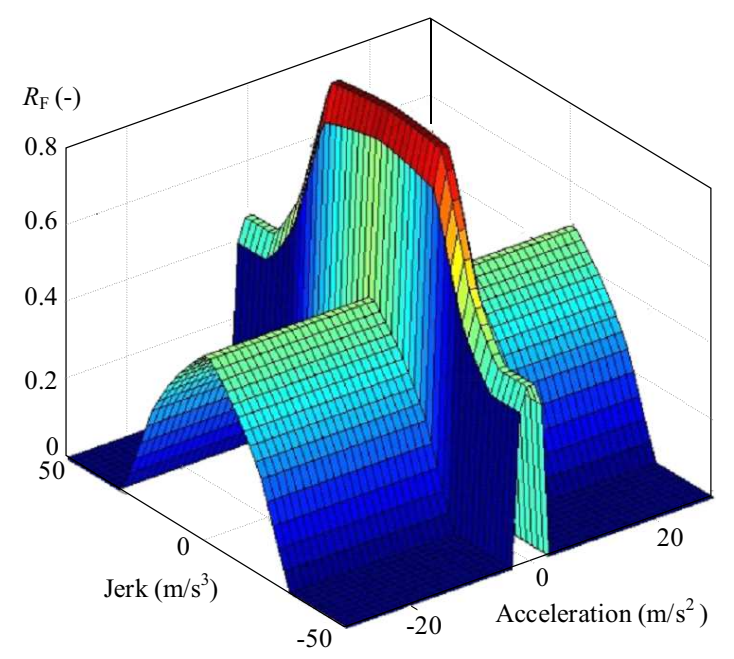

Fig. 7. Fuzzy logic surface

\section{Simulation results}

\subsection{Input data}

The simulations are based on measured data that was gained on a freight train. During the measurement were measured wheelsets velocities, tractive effort and a train velocity. Every of these values were calculated by train computer. The train velocity is calculated as minimum velocity from all wheelsets, and the velocity is filtered. Train velocity and the position were measured by GPS. The basic train parameters are shown in Table 1. 


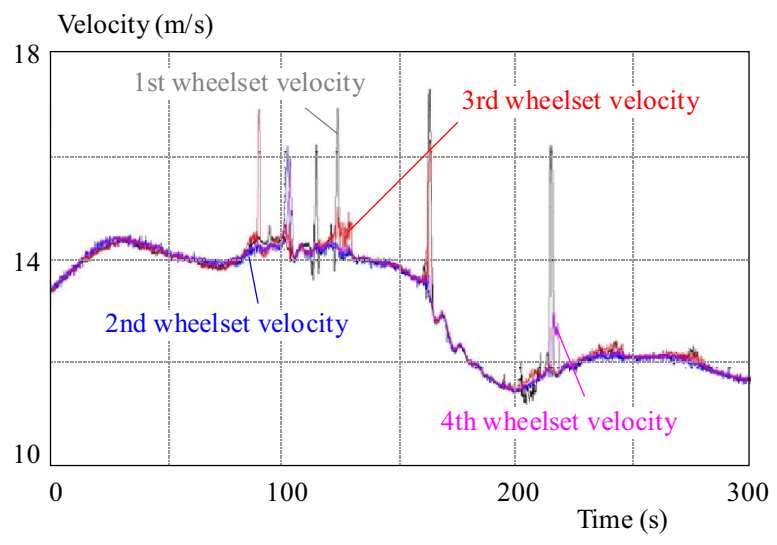

Fig. 8. Measured wheelsets velocity with high values of slip velocity

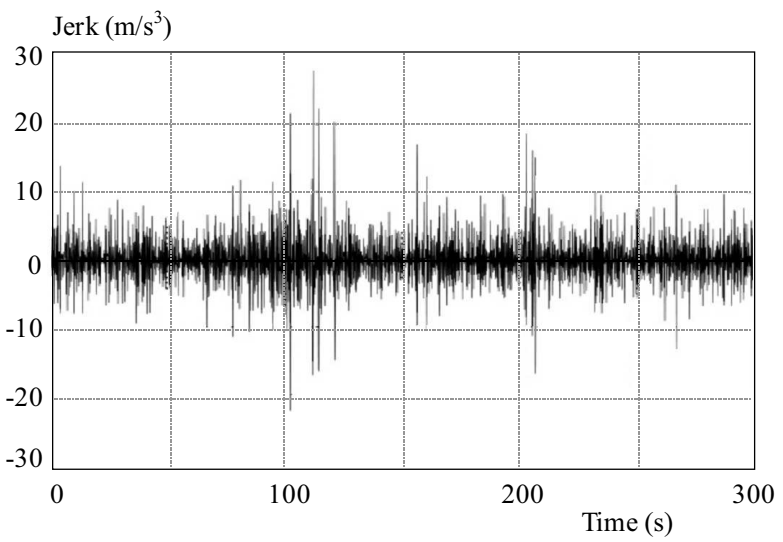

Fig. 10. Jerk calculated from minimal train velocity

Table 1. Train parameters

\begin{tabular}{lc}
\hline Name & Value \\
\hline Locomotive tractivve effort (maximal) & $360 \mathrm{kN}$ \\
Maximal track slope & $17 \%$ \\
rain weight (aproximatelly) & $1500 \mathrm{t}$ \\
Nominal wheel diameter & $1250 \mathrm{~mm}$ \\
\hline
\end{tabular}

The data were measured on the freight train on track with high slope track. Therefore, the high values of slip velocity occur during the measurement. The measured velocities of all wheelsets are shown in Fig. 8. There is also shown a part of measured data with multiple high values of the slip velocity caused by poor adhesion conditions and train mass during the train went to uphill. The high value of the slip velocity occurs on every wheelset, and the maximal value of the slip velocity was higher than $10 \mathrm{~km} / \mathrm{h}$. Every of the slip peak limitation required the re-adhesion controller act that abruptly decreased locomotive tractive effort applied on the wheelset.

In Fig. 9 and Fig. 10, there are shown calculated acceleration and jerk. Figure 9 and Fig. 10 correspond to Fig. 8 in the time period. The data shows part of the track where the locomotive hauls. The values are calculated from minimum velocity that is calculated from all wheelsets velocities. The high values of acceleration and

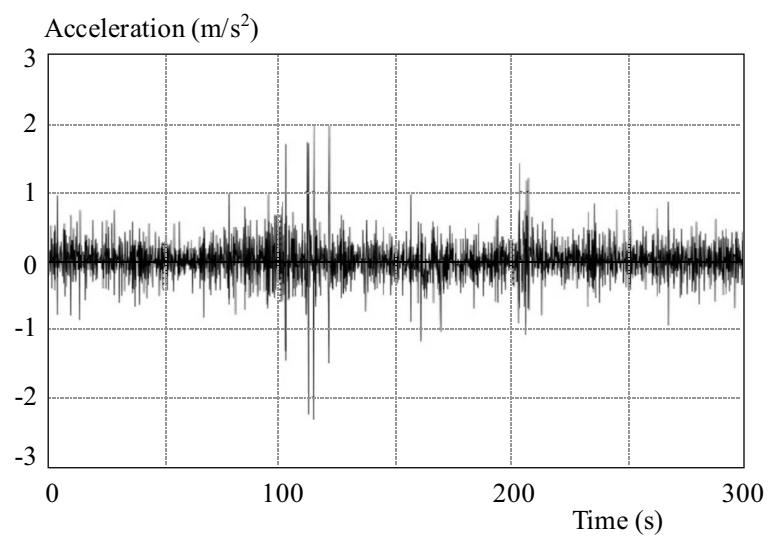

Fig. 9. Acceleration calculated from minimal train velocity

jerk are related to the high value of slip velocities. That is associated with high wheel acceleration, and the high value of the slip velocity is connected with low damping of the dynamic motions.

In Fig. 10, there is shown the importance of using jerk for fuzzy logic input. Acceleration values are more consistent in parts where the high values of the slip velocity do not occur as it is shown in Fig. 9 Therefore, it is difficult to determine between the low acceleration that corresponds to the train acceleration and the wheels acceleration. In Fig. 10 the signal is less consistent, and it is possible to determine the type of acceleration easier.

\subsection{Results}

There is shown the weighted coefficient output value in Fig. 11. It is clear that the limitation mainly occurs when the velocity has the high value of the slip, and the acceleration and jerk are the highest.

In Fig. 12, there is shown the calculated $R_{\mathrm{G}}$ weighting coefficient. The coefficient is calculated according to (5). The value corresponds to the train acceleration, and it is derived from the wheel acceleration and jerk. Comparing Fig. 12 with Fig. 9, it is clear that the calculated train acceleration is significantly limited. The limitation is done only by the fuzzy logic with no filter therefore without any additional time delay.

In Fig. 13, there is shown the train velocity calculated by the adaptive filter and the velocity is compared with velocity measured by GPS and measured wheelset velocity. The calculated train velocity is close to the GPS velocity that could be considered as the train velocity. The measurement was done on the locomotive with worn wheels. Therefore, the measured wheelset velocity is higher than its actual velocity. Therefore, the calculated train velocity by the adaptive filter should be higher than GPS velocity. The velocities are the same because the adaptive filter was tuned on the calculated velocity fit with GPS velocity for presentation purpose. The Fig. 13 shows that the wheel velocity is changed abruptly while the train velocity changes slowly. There are shown some differences between the filter output and the GPS. The main difference is shown when the high values of the slip 


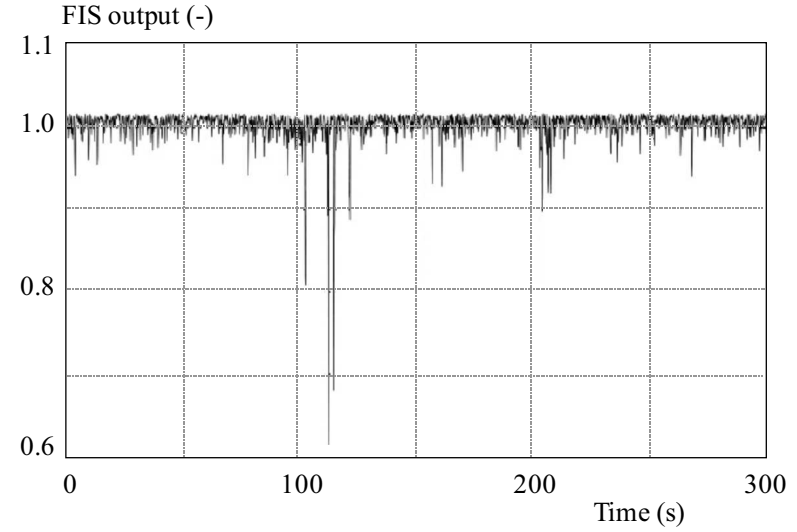

Fig. 11. Weighted coefficient (FIS output)

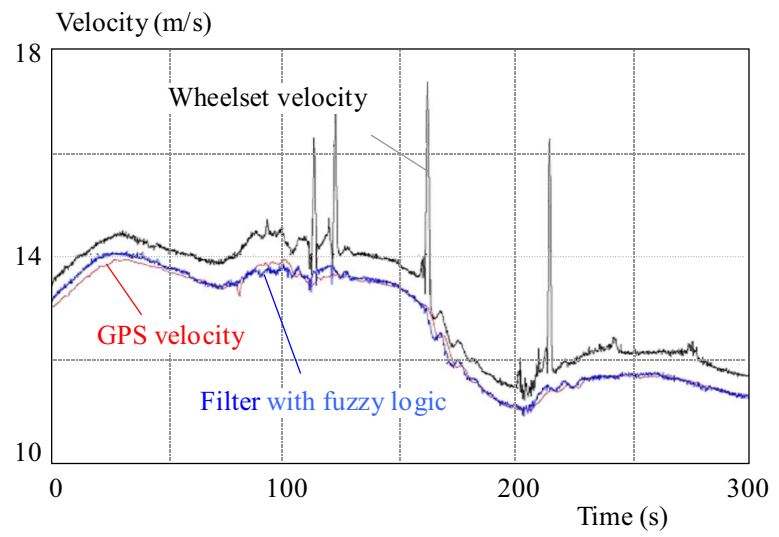

Fig. 13. Comparison of calculated velocity with GPS velocity and measured velocity

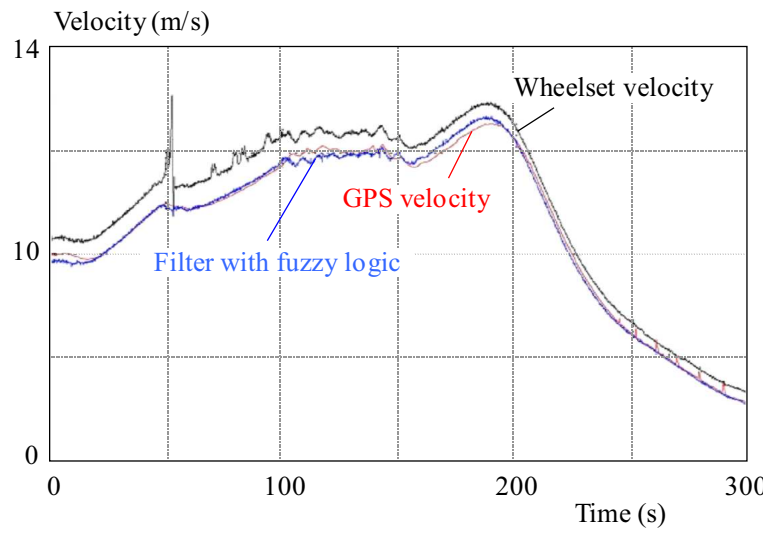

Fig. 15. Comparison of calculated velocity with GPS velocity and measured velocity

velocity occur. In this case, the filter output could be more rippled because the filter input data are rippled too.

The difference between the train velocity calculated by the adaptive filter and the GPS velocity shows Fig. 14. The slip velocity calculated by using a GPS velocity and the slip velocity calculated by the adaptive filter also shows Fig. 14. There is a slight difference between the velocities. The maximal difference is up to $0.30 \mathrm{~m} / \mathrm{s}$ that

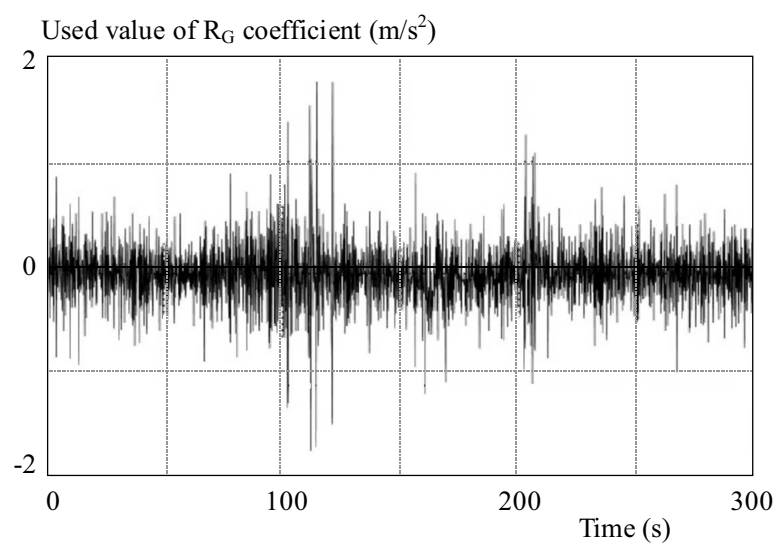

Fig. 12. Used value of $R_{\mathrm{G}}$ coefficient

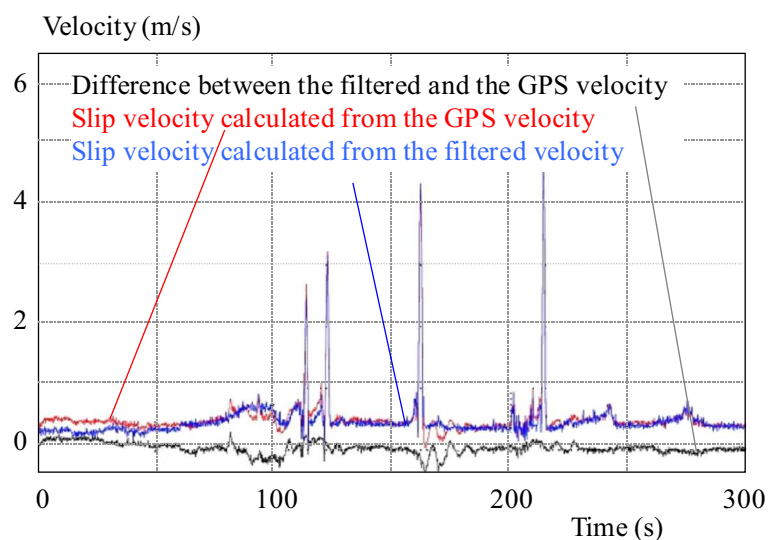

Fig. 14. Comparison between GPS velocity and the filtered velocity

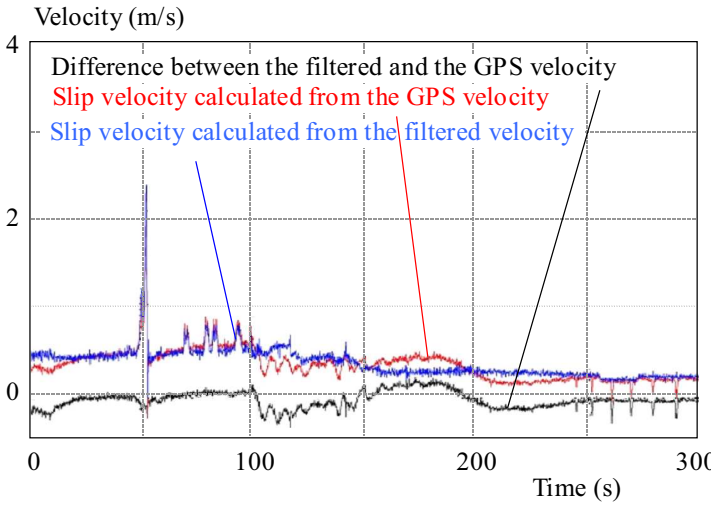

Fig. 16. Comparison between GPS velocity and the filtered velocity

represents $2 \%$ from the actual velocity. This accuracy can be considered as sufficient considering a GPS error.

Another example of filtered data shows Fig. 15. The figure shows locomotive hauling with a high value of the slip velocity and after time 190 seconds a locomotive run without tractive effort. The estimation is similar to the Fig. 13. In part without traction is the estimation close to velocity measured by the GPS. Corresponding 
slip velocity shows Fig. 16. The maximal difference is the same as in previous the example.

\section{Conclusion}

There is described there the adaptive filter that is tuned by the fuzzy logic in the paper. The fundamental principle of train velocity estimation is introduced. The difference between wheel circumference velocity and its longitudinal velocity is the main problem with estimating of the train velocity. The proposed train velocity estimation method is based on the adaptive filter. Its input is the minimum velocity of all driven wheelsets or the maximum velocity for braked wheelsets. The filter is tuned by the fuzzy logic. The wheelset acceleration and jerk are the fuzzy logic inputs. The fuzzy logic calculates the value of the weighted coefficient. From the coefficient is calculated value of the adjustment parameter of filter sensitivity $R_{\mathrm{G}}$. The simulation results show that the filter output is close to the velocity measured by the GPS that could be considered as the train velocity. The input data were measured on the locomotive that hauls a freight train.

\section{Acknowledgment}

This work was supported by the Grant Agency of the Czech Technical University in Prague, grant No. SGS16/083/OHK3/1T/13, Design of a Locomotive Slip Controller Based on Wheelset Torsional Vibration.

\section{REFERENCES}

[1] T. Atanabe, "Anti-slip Readhesion Control with Presumed Adhesion Force", Method of Presuming Adhesion Force and Running Test Results of High Speed Shinkansen Train, Quarterly Report of Railway Technical Research Institute, QR of RTRI, vol. 41, no. 1, (2000).

[2] S. H. Ark, J. S. Kim, J. J. Choi and H. Yamazaki, "Modeling and control of adhesion force railway rolling stocks", Control Systems, IEEE , vol. 28, no.5, (2008), 44-58.

[3] K. Ondo, "Anti-slip control technologies for the railway vehicle traction", Vehicle Power and Propulsion Conference (VPPC), 2012 IEEE, (2012), 1306-1311.

[4] K. Artani, M. Bourahla and Y. Miloud, "New AntiSkid Control For Electric Vehicle Using Behaviour Model Control Based On Energetic Macroscopic Representation", Journal of Electrical Engineering, Vol 59, 5 (2008) 225-233.

[5] F. Iang and Z. Gao, "An adaptive nonlinear filter approach to the vehicle velocity estimation for ABS", Control Applications 2000, Proceedings of the 2000 IEEE International Conference on, (2000), 490-495.

[6] L. Hu, L. Chao, L. Zhang and Y. Shi, "Design of longitudinal vehicle velocity observer using fuzzy logic and Kalman filter", Electronic and Mechanical Engineering and Information Technology
(EMEIT), 2011 International Conference on , vol. 6, no.(2011), 3225-3228.

[7] L. Hu, Y. Shi, Y. Zhang, H. Liu and M. Xu, "Vehicle lateral and longitudinal velocity estimation based on Adaptive Kalman Filter", Advanced Computer Theory and Engineering (ICACTE), 2010 3rd International Conference on , vol. 3, (2010), 325-329.

[8] M. Amashita and T. Watanbe, "A readhesion control method without speed sensor for electric railway vehicles", Electric Machines and Drives Conference, (IEMDC'03).IEEE International, vol. 1, (2003), 291-296.

[9] B. V, "Design of velocity radar for railway", Microwave and Millimeter Wave Technology (ICMMT), 2010 International Conference on, (2010), 1637-1639.

10] L. Uan, W. Zhao, C. Li and D. Zhou, "Error correction method for train speed measurement using Doppler radar train control system", Autonomous Decentralized Systems (ISADS), 2013 IEEE Eleventh International Symposium, (2013).

11] K. Im, S. Kong and S. Jeon, "Slip and Slide Detection and Adaptive Information Sharing Algorithms for High-Speed Train Navigation Systems", Intelligent Transportation Systems, IEEE Transactions on , vol. 16, no.6 (2015), 3193-3203.

12] M. Alvezzi, P. Toni, B. Allotta and V. Colla, "Train speed and position evaluation using wheel velocity measurements", Advanced Intelligent Mechatronics, 2001.Proceedings.2001 IEEE/ ASME International Conference on , vol. 1, (2001), 220-224.

[13] W. In, L. Diao, G. Zhang, D. Chen and Z. Li, "Maximum Adhesion Force Control Simulated Model of Electric Locomotive", Automation and Logistics, 2007 IEEE International Conference on, (2007), 1704-1708.

[14] K. Hishi, Y. Ogawa, I. Miyashita and S. Yasukawa, "Adhesion control of electric motor coach based on force control using disturbance observer", Advanced Motion Control, 2000, Proceedings, 6th International Workshop, (2000), 323-328.

[15] Y. Himizu, K. Ohishi, T. Sano, S. Yasukawa and T. Koseki, "Anti-slip re-adhesion control based on disturbance observer considering bogie vibration", Power Electronics and Applications, 2007 European Conference on , vol. , no., (2007), 1-10.

[16] M. Miri and B. Moaveni, "Vehicle velocity estimation based on data fusion by Kalman filtering for ABS", Electrical Engineering (ICEE), 2012 20th Iranian Conference on, (2012), 1495-1500.

Received 18 April 2016

Petr Pichlík received the master degree in electrical engineering from the Czech Technical University in Prague in 2011. He is currently he is PhD student and research engineer at Czech Technical University in Prague, Faculty of Electrical Engineering at the Department of Electric Drives and Traction. The theme of the thesis is slip control strategy for railway traction vehicles. His research interests simulation of vehicles electric drive and microcontrollers programming.

Jiří Zděnek received the master degree in electrical engineering from the Czech Technical University in Prague in 1974 and PhD degree in 1987. He is currently research worker in CTU in Prague, Faculty of Electrical Engineering, Department of Electric Drives and Traction. His research interests are electric traction vehicles and control computer hardware and software. 